\title{
Anisotropy of dark matter annihilation with respect to the Galactic plane
}

\author{
V S Berezinsky ${ }^{1}$, V I Dokuchaev ${ }^{2}$ and Yu N Eroshenko ${ }^{2}$ \\ ${ }^{1}$ INFN, Laboratori Nazionali del Gran Sasso, I-67010 Assergi (AQ), Italy \\ ${ }^{2}$ Institute for Nuclear Research of the Russian Academy of Sciences, Moscow, Russia \\ E-mail: dokuchaev@inr.npd.ac.ru
}

\begin{abstract}
We describe the anisotropy of dark matter clump distribution caused by tidal destruction of clumps in the Galactic disk. A tidal destruction of clumps with orbit planes near the disk plane occurs more efficiently as compared with destruction of clumps at near-polar orbits. A corresponding annihilation of dark matter particles in small-scale clumps produces the anisotropic gamma-ray signal with respect to the Galactic disk. This anisotropy is rather small, $\sim 9 \%$, and superimposed on that due to off-centering position of the Sun in the Galaxy. The anisotropy of annihilation signal with respect to the Galactic disk provides a possibility to discriminate dark matter annihilation from the diffuse gamma-ray backgrounds of other origin.
\end{abstract}

PACS numbers: 95.35.+d, 98.35.Gi, 95.30.Cq

Submitted to: JCAP 


\section{Introduction}

A primordial power-law spectrum of density fluctuations in the Dark Matter (DM) ranges from the largest scales above the scales of superclusters of galaxies to the smallest sub-stellar scales according to prediction of inflation models. This permits to predict the properties of smallest DM structures from the known CMB fluctuations at large scales. Substructures of DM in the galactic haloes with a rather large mass, $\geq 10^{7} M_{\odot}$, were extensively discussed in early works, see for example [1]. The nonlinear dynamics and mechanism of hierarchical clustering of these large DM clumps were analyzed in both analytical calculations [2, 3, 4] and numerical simulations [5, 6, 7]. At sub-stellar mass-scales of DM fluctuations, a principal new phenomenon arise - the cutoff of mass spectrum due to collisional and collisionless (free streaming) damping processes of DM particles in the forming clumps. The resulting smallest mass of DM clumps is determined by the properties of DM particles, in particular, by their elastic scattering. For detailed calculations of this cutoff see e. g. [8] and references therein. Additionally the cutoff of mass spectrum is influenced by the acoustic absorption [9] at the time of kinetic decoupling of DM particles [10] and also by the horizon-scale perturbation modes [11]. In [12] the kinetic equations for DM phase space density were solved in the case of perturbed cosmological background by taking into account the acoustic absorption, horizon-scale modes and gravitational perturbations. A corresponding value of the smallest clump mass for neutralino DM is of the order of the Moon or Earth mass.

The formation of small-scale DM clumps with a mass larger than the Earth mass, $M_{\text {min }} \sim 10^{-6} M_{\odot}$, have been explored in numerical simulations [13, 14]. A resulting differential number density of small-scale clumps, $n(M) d M \propto d M / M^{2}$, turns out very close to that obtained in the numerical simulations of large-scale clumps with mass

$M \geq 10^{6} M_{\odot}$. The other important result obtained in numerical simulations [13] is determination of the internal density profile in the isolated clump of minimal mass. The resulting density profile is approximately a power-law, $\propto r^{-\beta}$, with $\beta=1.5-2.0$, which is in a good agreement with theoretically predicted value $\beta=1.7-1.8$ according to [2].

The number density of small-scale DM clumps existing nowadays in the Galactic halo is determined by their tidal destruction during hierarchical structure formation [15] and also by tidal interactions with stars in the Galaxy [13, 16, 17, 18, 19, 20, 21]. Annihilation of DM particles in small-scale clumps [14, 22, 23, 24, 25, 26, 27, 28, 29] enhances the total DM annihilation signals in our Galaxy and thus boosts a chance for indirect detection of DM.

The usual assumption in calculations of DM annihilation is a spherical symmetry of the Galactic halo. In this case an anisotropy of annihilation gamma-radiation is only due to off-center position of the Sun in the Galaxy. Nevertheless, a principal significance of the halo nonsphericity for the observed annihilation signal was demonstrated in [30]. According to observations, the axes of the Galactic halo ellipsoid differ most probably no more than $10-20 \%$, but even a much more larger difference of axes, up to a factor 2 , can not be excluded [31, 32]. This leads to more than an order of magnitude uncertainty 
in the predicted annihilation flux from the Galactic anti-center direction [30]. It must be noted also the "intrinsic" annihilation anisotropy caused by the small-scale DM clustering itself. A corresponding angular power spectrum of annihilation signal at small scales is connected with a power spectrum of DM clumping [33]. In principle, the DM clumps may be seen as point sources at the gamma-sky [30]. Another minor source of annihilation anisotropy is a dipole anisotropy due to proper motion of the Sun in the Galaxy [34].

In [17] the anisotropy with respect to the Galactic disk was discussed basing on the numerical calculations of the destruction of DM clumps by stars in the disk and taking into account the influence of gravitational potential of the disk on the clump orbits. It was also shown [15, 19] that (i) small-scale DM clumps dominate in the generation of annihilation signal and (ii) the Galactic stellar disk provides the main contribution to the tidal destruction of clumps at $r>3 \mathrm{kpc}$, i. e. outside the central bulge region. A process of clump destruction in the halo is anisotropic in general (e. g. it depends on the inclination of clump orbit with respect to the disk plane). Respectively, the DM annihilation in the halo is also anisotropic. In this work we estimate the value of this anisotropy. It must be stressed that with a present state of art it is impossible to separate this source of anisotropy from that produced by the halo nonsphericity. More detailed investigation is required to constrain the shape of the halo and to search the distinctive features of annihilation anisotropy due to non-spherical halo clumpinesss. The detectors at the GLAST satellite will be sensitive to anisotropy up to $0.1 \%$ level [34. This will provide a hope to discriminate the anisotropic DM annihilation signal from the diffuse gamma-ray backgrounds.

\section{Destruction of clumps by disk}

Crossing the Galactic disk, a DM clump can be tidally destructed by the collective gravitational field of stars in the disk. This phenomenon is similar to the destruction of globular clusters by the "tidal shocking" in the Galactic disk [35]. The corresponding energy gain per unit mass of a clump at one disk crossing [35] is

$$
\Delta \tilde{E}=\frac{2 g_{m}^{2}(\Delta z)^{2}}{v_{z, c}^{2}}
$$

where $g_{m}$ is the maximum gravitational acceleration of the clump moving through the disk, $\Delta z$ is a vertical (perpendicular to the disk plane) distance of a DM particle from the clump center, $v_{z, c}$ is a vertical component of velocity at disk crossing. The dependence of $v_{z, c}$ on the inclination of orbit relative to the disk plane is the origin of the discussed anisotropy in the clump destruction, and, as a result, the origin of the anisotropy in annihilation signal.

The surface mass of the Galactic disk [36] can be approximated as

$$
\sigma_{s}(r)=\frac{M_{\mathrm{d}}}{2 \pi r_{0}^{2}} e^{-r / r_{0}}
$$


with $M_{\mathrm{d}}=8 \times 10^{10} M_{\odot}$ and $r_{0}=4.5 \mathrm{kpc}$, and therefore

$$
g_{m}(r)=2 \pi G \sigma_{s}(r) .
$$

We use the power-law parametrization [2, 3, 4, 13] of the internal density of a clump

$$
\rho_{\text {int }}(r)=\frac{3-\beta}{3} \rho\left(\frac{r}{R}\right)^{-\beta},
$$

where $\rho$ and $R$ are the mean internal density and a radius of clump, respectively, $\beta=1.8$ and $\rho_{\text {int }}(r)=0$ at $r>R$. The total (kinetic plus potential) internal energy of a clump for density profile (44) is given by

$$
|E|=\frac{3-\beta}{2(5-2 \beta)} \frac{G M^{2}}{R},
$$

where $M$ is the mass of the clump. Integrating (11) over a clump volume and using the density profile (41), one obtains an energy gain for the whole clump as

$$
\frac{\Delta E}{|E|}=\frac{(5-2 \beta)}{\pi(5-\beta)} \frac{g_{m}^{2}}{G \rho v_{z, c}^{2}} .
$$

We will use the following criterium for a tidal destruction of clump: a clump is destructed if a total energy gain $\sum \Delta E_{i}$ after several disk crossings exceeds the initial internal energy $|E|$ of a clump.

Let us consider now some particular orbit of a clump in the halo with an "inclination" angle $\gamma$ between the normal vectors of the disk plane and orbit plane. The orbit angular velocity at a distance $r$ from the Galactic center is $d \phi / d t=J /\left(m r^{2}\right)$, where $J$ is an orbital angular momentum of a clump. A vertical velocity of a clump crossing the disk is

$$
v_{z, c}=\frac{J}{m r_{\mathrm{c}}} \sin \gamma
$$

where $r_{\mathrm{c}}$ is a distance of crossing point from the Galaxy center. There are two crossing points (with different values of $r_{\mathrm{c}}$ ) during the one orbital period. The momentum approximation used here for calculations of the tidal heating is violated at small inclination angles, $\gamma \ll 1$. Nevertheless the resultant anisotropy is a cumulative quantity. It results from an integration over all clump orbits, and orbits with $\gamma \ll 1$ provide only small input into the anisotropy value.

We use here the Navarro-Frenk-White (NFW) density profile for the Galactic halo:

$$
\rho_{\mathrm{H}}(r)=\frac{\rho_{0}}{(r / L)(1+r / L)^{2}},
$$

where $\rho_{0}$ is normalized in such a way that at position of the Sun $\rho_{\mathrm{H}}\left(r_{\odot}\right)=0.3 \mathrm{GeV} / \mathrm{cm}^{3}$ and $L=45 \mathrm{kpc}$.

Let us introduce the following set of dimensionless variables:

$$
\begin{aligned}
& x=\frac{r}{L}, \quad \tilde{\rho}(x)=\frac{\rho_{\mathrm{H}}(r)}{\rho_{0}}, \quad y=\frac{J^{2}}{8 \pi G \rho_{0} L^{4} M^{2}}, \\
& \varepsilon=\frac{E_{\mathrm{orb}} / M-\Phi_{0}}{4 \pi G \rho_{0} L^{2}}, \quad \psi=\frac{\Phi-\Phi_{0}}{4 \pi G \rho_{0} L^{2}},
\end{aligned}
$$


where $\Phi_{0}=-4 \pi G \rho_{0} L^{2}, E_{\text {orb }}$ is an total orbital energy (kinetic and potential) of a clump. In these dimensionless variables the density profile of the halo (8) has a form

$$
\tilde{\rho}(x)=\frac{1}{x(1+x)^{2}} .
$$

Consider the Galactic halo model with an isotropic velocity distribution, which is appropriate for the halo formed by the hierarchial clustering of sub-haloes. In this model the distribution function depends only on energy. The gravitational potential $\psi(x)$, which corresponds to the density profile (11) is given by

$$
\psi(x)=1-\frac{\log (1+x)}{x} .
$$

The equation for the turning points of an orbit, $\dot{r}^{2}=0$, for the potential (12) can be written as

$$
1-\frac{\log (1+x)}{x}+\frac{y}{x^{2}}=\varepsilon
$$

From (13) one can find numerically the minimum $x_{\min }$ and maximum $x_{\max }$ distance between a clump and the Galactic center as function of $\varepsilon$ and $y$. Now denoting $p=\cos \theta$, where $\theta$ is an angle between the radius-vector $\vec{r}$ and the particle velocity $\vec{v}$, we have

$$
y=\left(1-p^{2}\right) x^{2}(\varepsilon-\psi(x)) .
$$

According to our assumption, the vectors $\vec{v} / v$ are distributed isotropically at each point $x$, and therefore $p$ has a uniform distribution in the interval $[0,1]$. The relation between $\tilde{\rho}(x)$ and the distribution function $F(\varepsilon)$ is given, according to [37], by

$$
\tilde{\rho}(x)=4 \pi \sqrt{2} \int_{\psi(x)}^{1} d \varepsilon[\varepsilon-\psi(x)]^{1 / 2} F(\varepsilon) .
$$

The function $F(\varepsilon)$ for a halo profile (111) can be fitted [38] with a good accuracy as

$$
F(\varepsilon)=F_{1}(1-\varepsilon)^{3 / 2} \varepsilon^{-5 / 2}\left[-\frac{\ln (1-\varepsilon)}{\varepsilon}\right]^{q} e^{P},
$$

where $F_{1}=9.1968 \times 10^{-2}, P=\sum_{i} p_{i}(1-\varepsilon)^{i},\left(q, p_{1}, p_{2}, p_{3}, p_{4}\right)=(-2.7419,0.3620,-$ $0.5639,-0.0859,-0.4912)$.

The time of motion from $x_{\min }$ to $x_{\max }$ and back is

$$
T_{\mathrm{c}}(x, \varepsilon, p)=\frac{1}{\sqrt{2 \pi G \rho_{0}}} \int_{x_{\min }}^{x_{\max }} \frac{d s}{\sqrt{\varepsilon-\psi(s)-y / s^{2}}},
$$

where $y$ depends on $x, \varepsilon$ and $p$ according to (14). The trajectory (the orbit) of a clump in the potential (12) is not closed. A precession angle during the time $T_{\mathrm{c}} / 2$ is

$$
\tilde{\phi}=y^{1 / 2} \int_{x_{\min }}^{x_{\max }} \frac{d s}{s^{2} \sqrt{\varepsilon-\psi(s)-y / s^{2}}}-\pi,
$$

and $\tilde{\phi}<0$. Therefore the orbital period is greater then $T_{\mathrm{c}}$ and equals to

$$
T_{\mathrm{t}}=T_{\mathrm{c}}(1+\tilde{\phi} / \pi)^{-1} \text {. }
$$


During a life-time of the Galaxy, $t_{\mathrm{G}} \simeq 10^{10} \mathrm{yrs}$, the relative total energy gain of a clump due to the tidal heating in multiple crossings of the disk is

$$
\frac{\Delta E}{|E|}=\frac{1}{|E|} \sum_{i=1}^{N}\left(\Delta E_{1}^{i}+\Delta E_{2}^{i}\right)
$$

where $\Delta E_{1}^{i}$ and $\Delta E_{2}^{i}$ are given by (6) for the two disk crossings during an orbital period $T_{\mathrm{t}}$, and $N \simeq t_{\mathrm{G}} / T_{\mathrm{t}}$. According to (17) the $z$-velocity component $v_{z, c} \propto x_{\mathrm{c}}^{-1}$, where $x_{\mathrm{c}}=r_{\mathrm{c}} / L$. Therefore one has to calculate the sum $\sum g_{m}^{2}\left(x_{\mathrm{c}}\right) x_{\mathrm{c}}^{2}$ with summation over all subsequent crossing points (odd and even) of a clump orbit with the Galactic disk. An important simplification in calculations follows from the fact that precession velocity is constant. For this reason the points of successive odd crossings are separated by

the same angles $\tilde{\phi}$. The same is also true for the successive even crossings. Using this simplification one can do the following transformation:

$$
\sum_{i=1}^{N} g_{m}(x)^{2} x^{2} \simeq \frac{1}{|\tilde{\phi}|} \int g_{m}(x)^{2} x^{2} d \phi \simeq \frac{1}{|\tilde{\phi}|} \int_{x_{\min }}^{x_{\max }} g_{m}(x)^{2} x^{2} \frac{d \phi}{d x} d x \frac{2 t_{\mathrm{G}}}{T_{\mathrm{t}}}
$$

where

$$
\frac{d \phi}{d x}=\frac{y^{1 / 2}}{x^{2} \sqrt{\varepsilon-\psi(x)-y / x^{2}}}
$$

is an equation of clump trajectory in the halo. Using the above formulae one finds

$$
\Delta E /|E|=\frac{A}{\sin ^{2} \gamma}
$$

where

$$
A(x, \varepsilon, y)=\frac{(5-2 \beta) t_{\mathrm{G}}}{2 \pi^{2}(5-\beta) G^{2} \rho \rho_{0} L^{2} y^{1 / 2}|\tilde{\phi}| T_{\mathrm{t}}} \int_{x_{\min }}^{x_{\max }} \frac{g_{m}^{2}(s) d s}{\sqrt{\varepsilon-\psi(s)-y / s^{2}}} .
$$

\section{Anisotropy of clump distribution}

A tidal heating and final destruction of clumps by the gravitational field of the Galactic disk depends on the inclination angle $\gamma$ of a clump orbit to the disk according to (1) and (23). This is a cause of the anisotropic clump number density decreasing during the lifetime of the Galaxy. A tidally induced anisotropy of clump distribution can be taken into account by inserting into the integral (15) an additional factor $e^{-\Delta E /|E|}=e^{-t_{\mathrm{G}} / t_{\mathrm{d}}}$, where $t_{\mathrm{d}}$ is an effective time of clump destruction. Due to an "anisotropy" factor $1 / \sin ^{2} \gamma$ in (23) the "tidal shocking" by the Galactic disk is most effective for clumps with orbit planes which are near coplanar to the disk plane (that is with an inclination $\gamma \ll 1$ ). Meanwhile for any intersection points there are many others orbits which pass through the disk plane with $\gamma \sim 1$. For this reason the resulting anisotropy in clump distribution is rather small. 


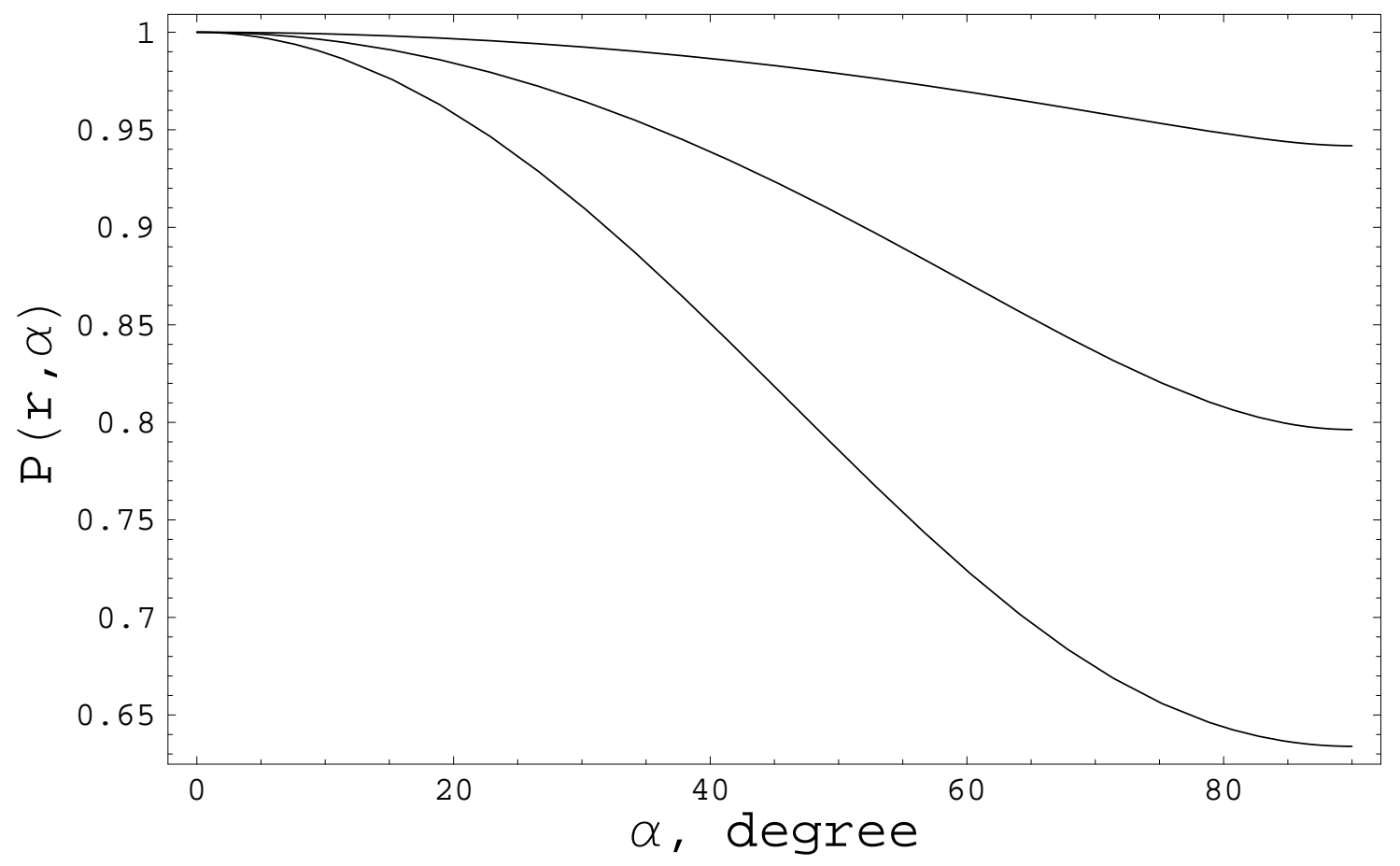

Figure 1. The normalized fractions of DM clumps in the halo $P(r, \alpha)$ from (25) which survive the tidal destruction by the stellar disk as a function of angle $\alpha$ between a radius-vector $\vec{r}$ and the disk polar axis. The plots are shown for radial distances from the Galactic center $r=3,8.5$ and $20 \mathrm{kpc}$ (from the bottom to the top). The curves must be multiplied by factors $0.04,0.4$ and 0.9 respectively to reproduce the actual values.

Let us consider a point in the halo with a radius vector $\vec{r}$ and an angle $\alpha$ with a polar axis of the disk. Only orbits with $\pi / 2-\alpha<\gamma<\pi / 2$ go through this point. A survival probability for clumps can be written now in the following form

$$
P(x, \alpha)=\frac{4 \pi \sqrt{2}}{\tilde{\rho}(x) \sin \alpha} \int_{0}^{1} d p \int_{0}^{\sin \alpha} d \cos \gamma \int_{\psi(x)}^{1} d \varepsilon[\varepsilon-\psi(x)]^{1 / 2} F(\varepsilon) e^{-\Delta E /|E|},
$$

where $\Delta E /|E|$ is defined by (23). This expression is derived from (15) by inserting the distribution over additional parameters $p$ and $\gamma$ (by taking in mind that we use the isotropic halo model) and the exponential factor for clumps destruction $e^{-\Delta E /|E|}$. The numerically calculated triple integral from (25) for survival probability $P(r, \alpha)$ is shown in the Fig. 1. The annihilation anisotropy is artificially enhanced in the Fig. 1 for better visualization for three chosen radial distances from the Galactic center, $r=3,8.5$ and $20 \mathrm{kpc}$ respectively by using the different multiplication factors. 


\section{Annihilation anisotropy}

For the diffuse distribution of DM in the halo, the annihilation signal (e. g. gamma-ray or neutrino flux per unit solid angle) is proportional to

$$
I_{\mathrm{H}}=\int_{0}^{r_{\max }(\zeta)} \rho_{\mathrm{H}}^{2}(\xi) d x,
$$

where $x=r / L$ and integration over $r$ goes along the line of sight, $\xi(\zeta, r)=\left(r^{2}+r_{\odot}^{2}-\right.$ $\left.2 r r_{\odot} \cos \zeta\right)^{1 / 2}$ is the distance to the Galactic center, $r_{\max }(\zeta)=\left(R_{\mathrm{H}}^{2}-r_{\odot}^{2} \sin ^{2} \zeta\right)^{1 / 2}+$ $r_{\odot} \cos \zeta$ is the distance to the external halo border, $\zeta$ is an angle between the line of observation and the direction to the Galactic center, $R_{\mathrm{H}}$ is a virial radius of the Galactic halo, $r_{\odot}=8.5 \mathrm{kpc}$ is the distance between the Sun and Galactic center. The corresponding signal from annihilations in DM clumps is proportional to the quantity [15]

$$
I_{\mathrm{cl}}=\mu S \rho \int_{0}^{r_{\max }(\zeta)} \rho_{\mathrm{H}}(\xi) P(\xi, \alpha) P_{\mathrm{sp}}(\xi) d x,
$$

where $\mu \simeq 0.05$ is a fraction of the DM mass in the form of clumps, $P_{\mathrm{sp}}$ is a survival probability of clumps due to their tidal destructions by stars in the halo and bulge from [19]. The function $S$ depends on the clump density profile and core radius of clump [15] and we use $S \simeq 14.5$. Here for simplicity we do not take into account the distribution of DM clumps over their internal densities. As a representative example we consider the Earth-mass clumps $M=10^{-6} M_{\odot}$ originated from $2 \sigma$ density peaks in the case of power-law index of primordial spectrum of perturbations $n_{p}=1$. The mean internal density of these clumps is $\rho \simeq 7 \times 10^{-23} \mathrm{~g} \mathrm{~cm}^{-3}$. The values of $\mu$ and $S$, as well as the distribution of the clumps over various parameters influence the annihilation signal but only weakly affect the predicted anisotropy.

In the Fig. 2 the annihilation signal calculated according to (27) is shown for the Galactic disk plane and for the orthogonal vertical plane (passing through the Galactic center) as function of angle $\zeta$ between the observation direction and the direction to the Galactic center. For comparison in the Fig. 2 is also shown the signal from the

spherically symmetric Galactic halo without the DM clumps (26). The later signal is the same in the in the Galactic disk plane and in vertical plane and therefore can be principally extracted from the observations.

The difference of the signals in two orthogonal planes at the same $\zeta$ can be considered as an anisotropy measure. Defined as $\delta=\left(I_{2}-I_{1}\right) / I_{1}$, it has a maximum value $\delta \simeq 0.09$ at $\zeta \simeq 39^{\circ}$.

\section{Discussions}

A total anisotropy of DM annihilation signal is determined in general by the Sun offcentering in the Galaxy and by the halo nonsphericity. An annihilation signal from 


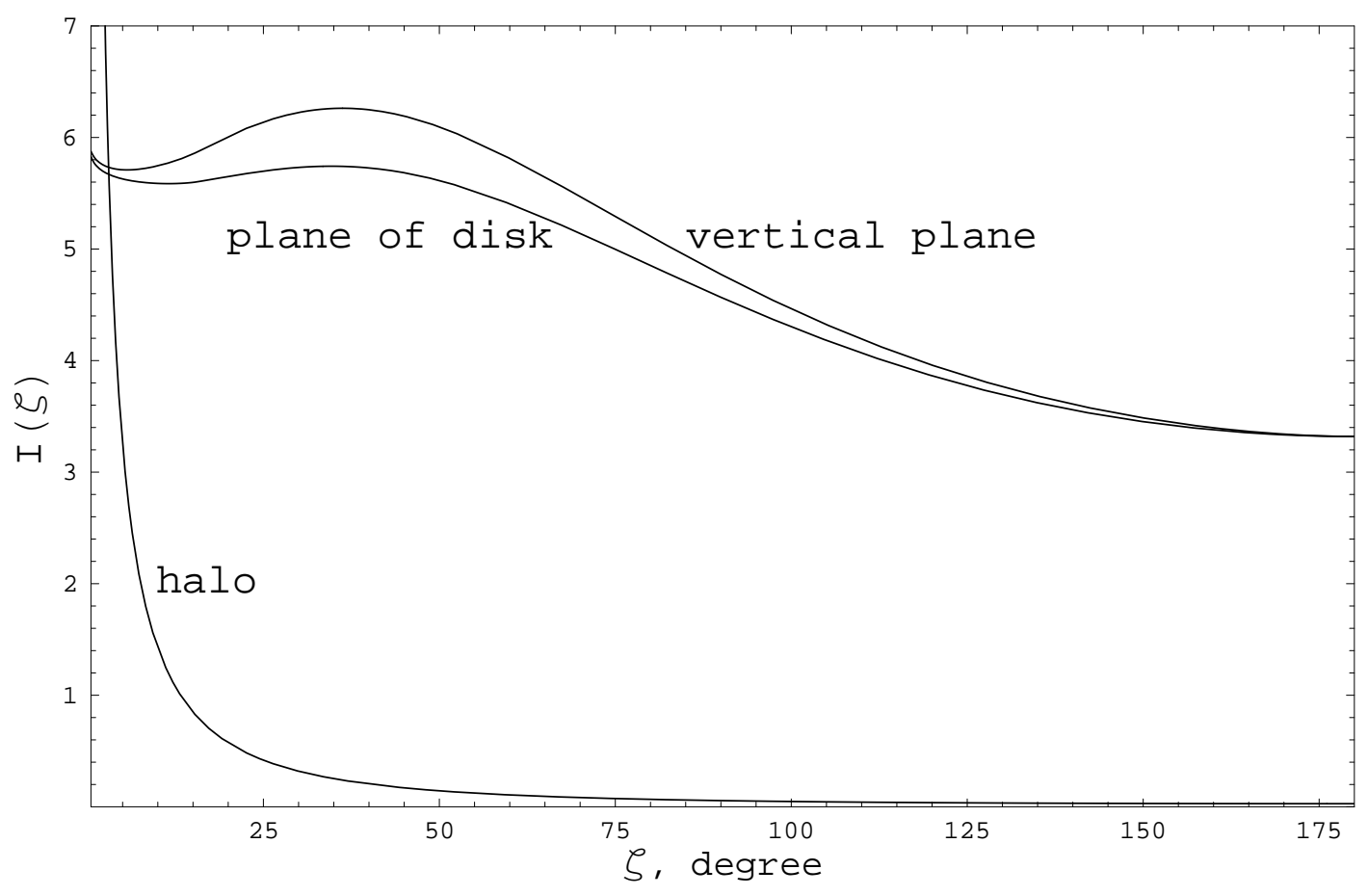

Figure 2. The annihilation signal (27) in the Galactic disk plane and in vertical plane as a function of the angle $\zeta$ between the line of observation and the direction to the Galactic center. For comparison it is shown also the annihilation signal from the Galactic halo without DM clumps (26). The values of both integrals (27) and (26) are multiplied by factor $10^{48}$.

the Galactic center depends on the central density profile of the Galactic halo. In the case with a density cusp [39], the bright source in the Galactic center is inevitable. Meanwhile, this cusp in the diffuse DM may be destroyed by the stellar feedback [40. The small-scale DM clumps are completely destructed inside the Galactic stellar bulge region. The "gamma-rings" are predicted in other galaxies due to the absence of clumps in their centers [17]. The unknown nonsphericity of the halo is a main source of anisotropy uncertainty. The value of anisotropy due to nonsphericity of the halo may be several times larger than one caused by the discussed in this paper effect of tidal destruction of DM clumps by the disk. More detailed analysis is required to separate these two sources of anisotropy. A nonsphericity (oblateness) of the halo due to the angular momentum can be easily estimated. It is natural to assume that the DM halo and disk have the same value of specific angular momentum (i. e. an angular momentum per unit mass). In this case the model of the Maclaurin spheroid for the halo gives only $\sim 0.5 \%$ difference for the halo axes. Therefore, the nonsphericity of the halo due to the angular momentum produces a negligible anisotropy.

In [17] the anisotropy with respect to disk plane in the Galaxy was pointed out. As it is seen from our calculations (see Fig. 2) this anisotropy of annihilation signal from the DM clumps with respect to the disk is rather small, $\sim 9 \%$, but far exceeds the anticipated GLAST resolution, $\sim 0.1 \%$. Therefore, the discussed anisotropy may be 
used in future detailed gamma-ray observations for discrimination of the annihilation signals from the DM clumps, diffuse DM in the Galactic halo and diffuse gamma-ray backgrounds.

In conclusion we list some unsolved problems and unclear features of DM clump physics. First of all, the detailed analytical theory of small DM clump clustering is desirable. An effective index of the density perturbation power spectrum $n \rightarrow-3$ at small-scales. This means that a gravitational clustering of small-scale structures proceeds very fast. As a result, the formation of small-scale DM clumps and their capturing by the larger ones are nearly simultaneous processes. The only approach developed to track this clustering is an approximate theoretical model [15, 19] and restricted numerical simulations [13]. During the past years the minimum clump mass is widely discussed. A value of the minimum mass has a principal significance for annihilation signal calculations. For this reason, the detailed calculations of minimum clump are requested. The central parts of DM clumps dominates in the generation of annihilation signal. For this reason the crucial problem is a value of the central density of DM clumps. To recover this value a detailed theoretical models and/or high-resolution numerical simulations are needed. The other problem is a survival of the central core of small-scale DM clump in tidal interactions. It is possible in principle that survived cores of DM clumps dominate in the annihilation signal. In this paper we evaluated only one particular part of the clump anisotropy in the halo. It would be useful also to clarify the halo shape and the nonsphericity of the distribution of clump orbits due to influence of the gravitational potential of the Galactic disk.

\section{Acknowledgments}

We thank Michael Kachelriess for useful discussion. This work was supported in part by the Russian Foundation for Basic Research grants 06-02-16029 and 06-02-16342, and the Russian President grants LSS 4407.2006.2 and LSS 5573.2006.2.

\section{References}

[1] Silk J and Stebbins A 1993 Astrophys. J. 411439

[2] Gurevich A V and Zybin K P 1988 Sov. Phys.-JETP 671

[3] Gurevich A V and Zybin K P 1988 Sov. Phys.-JETP 671957

[4] Gurevich A V and Zybin K P 1995 Sov. Phys.-Usp. 165723

[5] Navarro J F, Frenk C S and White S D M 1996 Astrophys. J. 462563

[6] Ghigna S, Moore B, Governato F, Lake G, Quinn T and Stadel J 2000 Astrophys. J. 544616

[7] Moore B et al. 1999 Astrophys. J. 524 L19

[8] Green A M Hofmann S and Schwarz D J 2005 JCAP 0508003

[9] Weinberg S 1971 Astrophys. J. 168175

[10] Hofmann S, Schwarz D J and Stocker H 2001 Phys. Rev. D 64083507

[11] Loeb A and Zaldarriaga M 2005 Phys. Rev. D 71103520

[12] Bertschinger E 2006 Phys. Rev. D 74063509

[13] Diemand J, Moore B and Stadel J 2005 Nature 433389

[14] Diemand J, Kuhlen M and Madau P 2006 Astrophys. J. 649 1; ibid. 2007 Astrophys. J. 657262 
[15] Berezinsky V, Dokuchaev V and Eroshenko Yu 2003 Phys. Rev. D 68103003

[16] Zhao H S, Taylor J, Silk J and Hooper D 2005 Earth-mass dark halos are torn into dark ministreams by stars Preprint astro-ph/0502049

[17] Zhao H S, Taylor J, Silk J and Hooper D 2007 Astrophys. J. 654697

[18] Moore B, Diemand J, Stadel J and Quinn T 2005 On the survival and disruption of Earth mass CDM micro-haloes Preprint astro-ph/0502213

[19] Berezinsky V, Dokuchaev V and Eroshenko Yu 2006 Phys. Rev. D 73063504

[20] Green A M and Goodwin S P 2007 Mon. Not. Roy. Astron. Soc. 3751111

[21] Angus G W and Zhao H S 2006 Analysis of galactic tides and stars on CDM microhalos Preprint astro-ph/0608580

[22] Berezinsky V, Bottino A and Mignola G 1997 Phys. Lett. B 391355

[23] Aloisio R, Blasi P and Olinto A V 2004 Astrophys. J. 60147

[24] Tasitsiomi A and Olinto A V 2002 Phys. Rev. D 66083006

[25] Taylor J E and Silk J 2003 Mon. Not. Roy. Astron. Soc. 339505

[26] Bergstrom L, Edsjo J and Ulio P 1998 Phys. Rev. D 58083507

[27] Bergstrom L, Edsjo J and Gunnarsson C 2001 Phys. Rev. D 63083515

[28] Ullio P, Bergstrom L, Edsjo J and Lacey C 2002 Phys. Rev. D 66123502

[29] Oda T, Totani T and Nagashima M 2005 Astrophys. J. 633 L65

[30] Calcaneo-Roldan and Moore B 2000 Phys. Rev. D 62123005

[31] Olling R P and Merrifield M R 2000 Mon. Not. Roy. Astron. Soc. 311361

[32] Olling R P and Merrifield M R 2001 Mon. Not. Roy. Astron. Soc. 326164

[33] Ando S and Komatsu E 2006 Phys. Rev. D 73023521

[34] Hooper D and Serpico P D 2007 Angular Signatures of Dark Matter in the Diffuse Gamma Ray Spectrum Preprint astro-ph/0702328

[35] Ostriker J P, Spitzer L Jr and Chevalier R A 1972 Astrophys. J. Lett. 17651

[36] Marochnik L S and Suchkov A A 1984 Galaxy (Moscow: Nauka)

[37] Eddington A S 1916 Mon. Not. Roy. Astron. Soc. 76572

[38] Widrow L M 2000 Astrophys. J. Supp. 13139

[39] Berezinsky V S, Gurevich A V and Zybin K P 1992 Phys. Lett. B 294221

[40] Mashchenko S, Couchman H M P and Wadsley J 2005 Nature 442539 